\title{
Impulse-Based Scheme for Crystal-Less ULP Radios
}

Salvatore Drago, Fabio Sebastiano, Lucien J. Breems, Senior Member, IEEE, Domine M. W. Leenaerts, Fellow, IEEE, Kofi A. A. Makinwa, Senior Member, IEEE, and Bram Nauta, Fellow, IEEE

\begin{abstract}
This study describes a method of implementing a fully integrated ultra-low-power (ULP) radio for wireless sensor networks (WSNs). This is achieved using an ad hoc modulation scheme (impulse radio), with a bandwidth of $17.7 \mathrm{MHz}$ in the $2.4 \mathrm{GHz}$-ISM band and a specific medium access control (MAC) protocol, based on a duty-cycled wake-up radio and a crystal-less clock generator. It is shown that the total average power consumption is expected to be less than $100 \mu \mathrm{W}$ with a clock generator inaccuracy of only $1 \%$.
\end{abstract}

Index Terms-Crystal-less clock, impulse radio, ultra-low power (ULP), wake-up radio, wireless sensor network (WSN), EDICS Category: COMM110A5, COMM200, COMM250A5.

\section{INTRODUCTION}

$\mathbf{R}$ ECENTLY, visions of a future in which technology is embedded, hidden, and adaptive to users have been proposed [1]. To implement such visions, distributed technology is required that automatically adapts to its environment without any user intervention. This in turn requires the existence of a hidden wireless infrastructure that is energy-autonomous and is able to transport a wide range of data types and content. A natural approach to realize such a scenario is by the use of wireless sensor networks (WSNs). These consist of thousands of ubiquitous nodes characterized by their small size and by their ability to collect and share data from the environment. Each of these nodes must be a completely autonomous system, comprising a sensor module, some (digital) processing capabilities, a radio interface, and a power source [2]. In the past, much effort has addressed the challenge of implementing WSN on a large scale, from high-level medium access control (MAC) protocols to electronic circuits [1].

The requirements for a WSN are diverse: in particular, ultralow-power (ULP) operation must be combined with small size and extremely low cost. Since battery replacement is impractical and expensive, the energy autonomy of each node is a strong requirement: the power consumption of each node should be sufficiently low to enable a lifetime of a few years while relying on a small battery or on "scavenged" energy from the environment. From literature, state-of-the-art transceivers consume at least an

Manuscript received September 29, 2008; revised December 07, 2008. First published February 13, 2009; current version published May 20, 2009. This work was supported by the European Commission through the Marie Curie Project TRANDSSAT-2005-020461. This paper was recommended by Guest Editor W. A. Serdijn.

S. Drago, F. Sebastiano, L. J. Breems, and D. M. W. Leenaerts are with the NXP Semiconductors, 5656AE Eindhoven, The Netherlands (e-mail: fabio. sebastiano@nxp.com; salvatore.drago@nxp.com).

K. A. A. Makinwa is with the Electronic Instrumentation Laboratory, Delft University of Technology, 2628CD Delft, The Netherlands.

B. Nauta is with the IC Design Group, CTIT Research Institute, University of Twente, 7500AE Enschede, The Netherlands.

Digital Object Identifier 10.1109/TCSI.2009.2015208 order of magnitude more energy than that delivered by most energy scavengers [3]. Another challenge is the need to integrate the whole node in silicon, so as to reduce both cost and size. Ideally, the antenna should be the only external component, as steps are being undertaken to integrate energy scavengers on chip [4].

In order to reduce its energy consumption, a unique characteristic of WSN can be exploited, i.e., the small amount of transmitted data: in periods ranging from seconds to some minutes, a typical node sends and receives packets of several hundreds of bits. Consequently, the largest fraction of its energy consumption is spent in idle listening to the channel, waiting for data packets [5]. Reduction of the energy wasted in idle listening is usually obtained by duty-cycling the network nodes, i.e., by putting them into a sleep mode for a significant fraction of the time. This task requires a synchronization algorithm to ensure that all nodes observe simultaneous sleep and wake-up times. Previous solutions have focused on the use of a reactive radio [6] or on a synchronous network protocol [7]. In the latter, the nodes are equipped with a high-accuracy clock which allows them to synchronize themselves in time. Each node can agree to use specific communication timeslots and can then be duty-cycled in order to listen to the channel only during these timeslots, thereby reducing its idle listening time. However, this solution requires an accurate timing reference, usually based on a quartz-crystal or a resonator, which are large and expensive and can hardly be integrated. In the reactive radio approach, nodes, in addition to a main radio for data communication, are equipped with a low-power wake-up radio, typically employing a very simple architecture based on high- $Q$ off-chip RF filters [8], [9]. However, no wake-up radio has yet been proposed both with sufficiently low power consumption and with acceptable sensitivity. In the wake-up approach, wasted energy is reduced by the introduction of an extra radio, while in the synchronous approach wasted energy is reduced by shortening the listening time. In both cases, external components, e.g., frequency references or high- $Q$ filters, are required, which directly increases cost and reduces the potential for full integration. Moreover, an accurate frequency reference is also needed to satisfy spectrum regulations and to tune the receiver to the incoming signal. This usually is achieved by using a crystal oscillator or, alternatively, by using a MEMS-based [9] or SAW-based transceiver [8].

These two points, i.e., reduced power consumption and complete integration in silicon, are analyzed in this study. Particular attention will be paid to the RF communication block, which is usually the bottleneck in WSN design because of its comparatively high power consumption [5]. It is the aim of this paper to consider these two points from application, MAC protocol, and circuit perspectives. A promising new low-power technique is proposed that enables the complete integration of a WSN node. It enables the realization of a crystal-less node by combining 
a duty-cycled wake-up radio and an impulse-based modulation scheme.

Starting from state-of-the-art and the application requirements (Section II), a simple MAC protocol is derived and presented in Section III; the modulation scheme and its influence on both the main receiver and the wake-up radio are described in detail respectively in Sections IV and V. Conclusions are drawn in Section VI.

\section{APPLICATION REQUIREMENTS}

WSNs can address a wide range of applications. Examples are the control of heating, ventilating, and air conditioning (HVAC) systems in buildings, habitat monitoring, precision agriculture, the monitoring of vital signs, and the control of food during processing and storage [10].

As a simple case, we consider a network containing a large number of low-power wireless sensor nodes and a minimum of one high-power data sink node. Data are spread over the network through multihop routing and are collected in the data sink for further use by a central control system. We assume that the data sink is able to receive data in the WSN format and does not affect the power consumption of the network-for this reason, it is not taken into account in the following. The network traffic is inherently low, since environmental parameters are generally slowly varying and can be sensed at low rates by the nodes. Moreover, if data redundancy (for example, due to the spatial vicinity of nodes sampling the same parameter) is reduced by aggregating data, the number of transmissions can be decreased [11]. Requirements on the quality of service (QoS), such as a maximum packet latency, are not specified, since the proposed system is intended for nontime-critical applications.

In order to make these networks a reality, the node's hardware implementation should meet four main requirements:

- small form factor (less than $1 \mathrm{~cm}^{3}$ ): a node should be placed and hidden in the environment;

- low cost: the number of external components must be minimized; when integrated energy scavengers are employed or batteries are embedded in the chip package, the only allowed external component is the antenna;

- energy autonomy: taking into account a lifetime of some years, battery capacity, state-of-the-art energy scavenging techniques and extrapolating their future developments, the power consumption of the whole node must be limited to $100 \mu \mathrm{W}$;

- wireless link: each node must be able to receive and transmit a small number of packets (less than 10) in a relatively long time span $(10 \mathrm{~min})$. The payload of each packet is of the order of $100 \mathrm{~b}$.

Application requirements have been derived from these considerations and are summarized in Table I. A system that satisfies such requirements can be used for most of the intended applications. Note that a bit error rate (BER) of $10^{-3}$ is sufficiently low to give a packet error rate lower than $10 \%$ (for a payload of $100 \mathrm{~b}$ ); this is an acceptable level of performance for the applications described, which are characterized by a very low average packet rate. Since the power in WSNs is not dominated by data transmission and reception, high packet error rate
TABLE I

APPLICATION REQUIREMENTS

\begin{tabular}{|l|l|}
\hline Number of nodes $(n)$ & 100 \\
\hline Maximum distance between two nodes $(d)$ & $10 \mathrm{~m}$ \\
\hline Packet rate $(P R)$ & $1 \mathrm{pkt} / \mathrm{min}$ \\
\hline Packet payload $\left(N_{p l}\right)$ & 100 \\
\hline Bit error rate $(B E R)$ & $10^{-3}$ \\
\hline Average power dissipation & $\leq 100 \mu \mathrm{W}$ \\
\hline
\end{tabular}

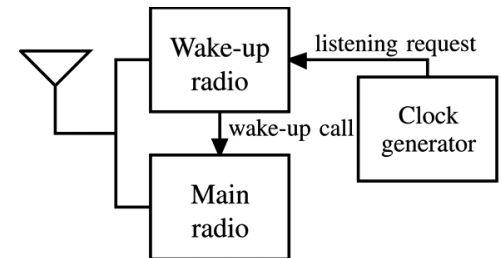

(a)

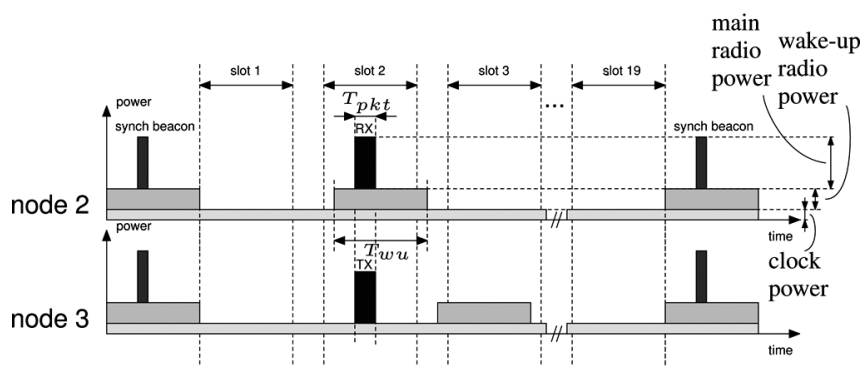

(b)

Fig. 1. (a) System architecture and (b) MAC protocol with energy breakdown.

can be tolerated, because packet retransmissions do not significantly affect the power consumption.

\section{MAC PROTOCOL: DUTY-CYCLED WAKE-UP RADIO}

\section{A. Principle of Operation}

In the proposed scheme, the receiver, while residing in a reduced power mode (sleep mode), is able to decide when to turn itself on to listen for communications (listening mode), and when communications are present, to prompt a full power-up of the device (communication mode). This can be implemented by the architecture in Fig. 1(a), comprising a wake-up radio responsible for monitoring the channel while waiting for data packets, a main radio to communicate, and a clock generator for synchronization. The wake-up radio is duty-cycled, i.e., put in listening mode on a scheduled basis by the clock generator to save energy when monitoring is not required. When a data packet is present, the wake-up radio triggers a wake-up call for the main radio. As shown in Fig. 1(b), time is divided into fixed slots, e.g., Slot 1, Slot 2, and so on, which form the basis of a time-division multiple access (TDMA) protocol. Accordingly, in each timeslot, only the wake-up radio of a particular node should be monitoring the channel. For example, Slot 2 can be allocated to Node 2, Slot 3 to Node 3, and so on. Any node can transmit a packet in any slot, depending on the intended recipient node of the data packet. A back-off algorithm handles collisions due to simultaneous transmission of packets from different sources to the same destination. In the figure, Node 3 sends a packet to Node 2 in Slot 2 . With this approach, the 
wake-up radio is active when a packet is expected, while the main radio is active only when a packet is effectively going to be received. Specialized timeslots are needed for the time synchronization of the whole network. In these dedicated timeslots, a particular node, called the master, sends special packets, labeled in Fig. 1(b) as "synch beacons." All nodes wake up to listen to the synchronization beacons and reset their internal clock on reception of the beacon. In the following, we assume that a synchronization beacon has the same structure of a normal packet.

If more reliability is required, the receiver can send an "acknowledgment" when a data packet is successfully received. The acknowledgment for a packet sent by a transmitting node $T$ to a receiving node $R$ can be either sent as a stand-alone data packet in the next available timeslot for $T$ or embedded in the payload of the next data packet from $R$ to $T$. Those approaches can increase the latency in the transmission of information, but it can be tolerated in the applications of interest in this work, which do not have stringent requirements on QoS. In any case, the use of acknowledgments affects the power consumption only marginally, since, as it will be shown in the following sections, the power is not dominated by data packet transmission and reception.

In the next section, it will be shown that the power consumption needed for an implementation of a TDMA scheme depends on the clock accuracy. The adoption of a TDMA scheme in combination with a duty-cycled wake-up radio reduces the power consumption for a given clock accuracy and consequently makes it possible to use a fully integrated clock with moderate accuracy, i.e., 5000 ppm or less. Moreover, since the power budget of the wake-up radio is increased by duty-cycling, its sensitivity can be increased without the use of off-chip filters.

\section{B. Optimization of MAC Performance}

The average power consumption of each node depends both on the power consumption of each single block (main radio, wake-up radio, and clock generator) and their duty-cycle factors. To determine the latter, it is necessary to determine the parameters of the MAC protocol, such as the duration of a timeslot and the rate of synchronization beacons, and then optimize the duty-cycle factors for the minimum power consumption.

The duration of the listening timeslots $\left(T_{\mathrm{wu}}\right)$ must be long enough to account for timing errors between the clocks of the receiver and the transmitter, thus ensuring that a packet is only transmitted when the recipient is in listening mode. Since the clocks are reset by the synchronization beacons, timing errors in receivers and transmitters are accumulated from the last beacon and depend on clock accuracy. We assume that the timing error of each clock is bounded by the interval $\left[-a_{\text {clock }} t,+a_{\text {clock }} t\right]$, where $a_{\text {clock }}$ is the relative clock accuracy and $t$ is the time that has elapsed since the last synchronization. Taking into consideration the fact that $T_{\mathrm{wu}}$ must comprise the time needed to receive at least $\alpha$ packets and a margin to account for the timing error, the following condition must hold:

$$
T_{\mathrm{wu}} \geq \frac{4 a_{\text {clock }}}{\mathrm{BR}}+\alpha T_{\mathrm{pkt}}
$$

where $a_{\text {clock }}$ is the relative clock accuracy, BR is the beaconing rate, i.e., the repetition rate of the synchronization beacons, $T_{\mathrm{pkt}}$ is the packet transmission time, and $\alpha$ is the number of packets that can be received in one slot. ${ }^{1}$ The factor 4 in (1) can be understood by considering the two worst cases: if the transmitter is early (late) by $a_{\text {clock }} / \mathrm{BR}$ and the receiver is late (early) by the same amount, the wake-up radio must be on at $2 a_{\text {clock }} / \mathrm{BR}$ before (after) the time expected according to the receiver clock.

However, $T_{\mathrm{wu}}$ cannot be arbitrarily large, as the protocol must assure the availability of a timeslot for each node. This is expressed by the following condition:

$$
\frac{1}{P R} \geq\left(n+\frac{B R}{P R}\right)\left(T_{\mathrm{wu}}+T_{\text {guard }}\right)
$$

where $P R$ is the packet rate of each node, $n$ is the number of nodes in the network, and $T_{\text {guard }}$ is the guard time between two successive timeslots. Equation (2) ensures that the interval $1 / P R$ is sufficiently wide to accommodate timeslots for reception of data packets and synchronization beacons.

The packet transmission time can be expressed as $T_{\mathrm{pkt}}=$ $\left(N_{\mathrm{pr}}+N_{\mathrm{pl}}\right) / D R$, where $N_{\mathrm{pr}}$ and $N_{\mathrm{pl}}$ are, respectively, the number of bits in the preamble and in the payload of each packet, and $D R$ is the data rate of the main radio. A high data rate would reduce the packet transmission time and consequently the duty-cycle of the main radio; however, power-hungry transceivers would be required to achieve very high data rates. On the other hand, low data rates and long transmission times can also be inefficient because in these cases the power in the transceiver would be dominated by data-rate independent blocks, such as RF oscillators. As a good tradeoff, $D R=100 \mathrm{kbps}$ is chosen [12].

The duty-cycle of the wake-up radio and of the transmitting and receiving sections of the main radio can be expressed, respectively, as

$$
\begin{aligned}
D C_{\mathrm{wu}} & =T_{\mathrm{wu}}(P R+B R) \\
D C_{r x} & =\frac{N_{\mathrm{pr}}+N_{\mathrm{pl}}}{D R}(P R+B R) \\
D C_{t x} & =\frac{N_{\mathrm{pr}}+N_{\mathrm{pl}}}{\mathrm{DR}} \mathrm{PR} .
\end{aligned}
$$

The average node power consumption is then defined as

$$
P=D C_{\mathrm{wu}} P_{\mathrm{wu}}+D C_{r x} P_{r x}+D C_{t x} P_{t x}+D C_{\mathrm{clk}} P_{\mathrm{clk}}
$$

where $P_{x}$ is the peak power consumption of block $x$ and it is assumed that $D C_{\text {clk }}=100 \%$ for the duty-cycle of the clock generator.

Using the previous equations, it is possible to find the optimum $B R$ which minimizes the power consumption and satisfies conditions (1) and (2). The results are shown in Fig. 2, in which the optimum BR and the minimum power consumption for the radio section (wake-up radio and main radio) are plotted as a function of the clock accuracy. We have assumed $P_{\mathrm{wu}}=500 \mu \mathrm{W}, P_{r x}=P_{t x}=2 \mathrm{~mW}$,

${ }^{1}$ Equation (1) gives a sufficient condition for enabling communication among all nodes. However, nodes whose timeslots occur shortly after a synchronization beacon can, in principle, have a shorter $T_{\text {wu }}$ and, consequently, a lower power consumption. This case is not analyzed in this study. In the following, it is implicitly assumed that $T_{\mathrm{wu}}$ is the same for all nodes. 


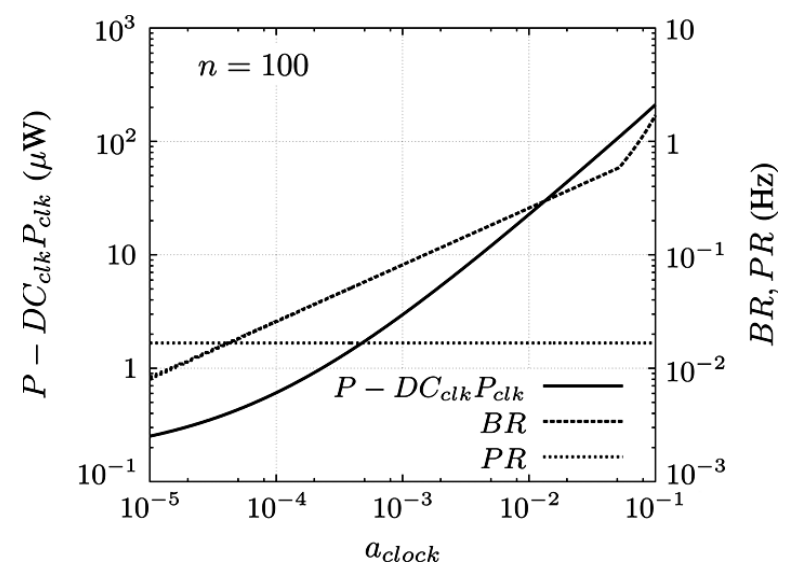

Fig. 2. Minimum average power consumption of the radio section (wake-up radio and main radio) and relative beacon rate versus clock accuracy for a network of 100 nodes; for reference, the packet rate is also plotted.

$P R=1 \mathrm{pkt} / \mathrm{min}, N_{\mathrm{pr}}=N_{p l}=100, T_{\text {guard }}=0.25 \cdot T_{\mathrm{wu}}$, and $\alpha=1$ in (1). These assumptions will be justified in the following sections. The power dissipation of the clock generator is not included in this analysis, as it was not possible to find a simple relation between its accuracy and its power consumption. Further considerations on a practical implementation are given in Section III-D. Fig. 2 shows that the power increases as the clock accuracy decreases. This can be expected as low values of $a_{\text {clock }}$ imply either longer timeslots or more frequent beacons. $B R$ is then optimized for the best balance between power spent in the wake-up radio, in the case of longer timeslots, and the power spent in the whole radio section in the case of more beacons. Network synchronization plays a crucial role in the estimation of the total power budget, and, in most cases, it also represents the dominant fraction of the power for this simple MAC scheme. In Fig. 2, $B R$ is higher than $P R$ for $a_{\text {clock }}$ higher than $40 \mathrm{ppm}$, which means that nodes are receiving beacons more often than data packets. It would then be more efficient to have a highly accurate clock; however, the analysis in this section shows that a power consumption compatible with system specifications is achieved also with relaxed accuracy and, consequently, with a fully integrated solution.

A discontinuity of the derivative of $B R$ is observable in Fig. 2. This can be explained by the fact that for high values of $a_{\text {clock }}$, very frequent beacons are needed to keep $T_{\text {wu }}$ sufficiently low to assign a timeslot to each node [see (1)]. The minimum power for the radio section and the network activity are plotted versus the number of nodes in Fig. 3. The activity of the network is defined as the ratio of the time during which at least one node is listening or receiving (comprising also the guard time) and the total available time. As $n$ increases, the activity saturates to $100 \%$. The average power also rises until a limit in the number of nodes is reached for which is not possible to satisfy conditions (1) and (2). This limit in the maximum number of nodes is clearly dependent on the accuracy of the timing reference and represents a limit to the scalability of the network. Moreover, the computations have been performed with a simple model where no errors, such as packet collisions, have been considered. We can take collisions into account by reserving time for the reception of more packets

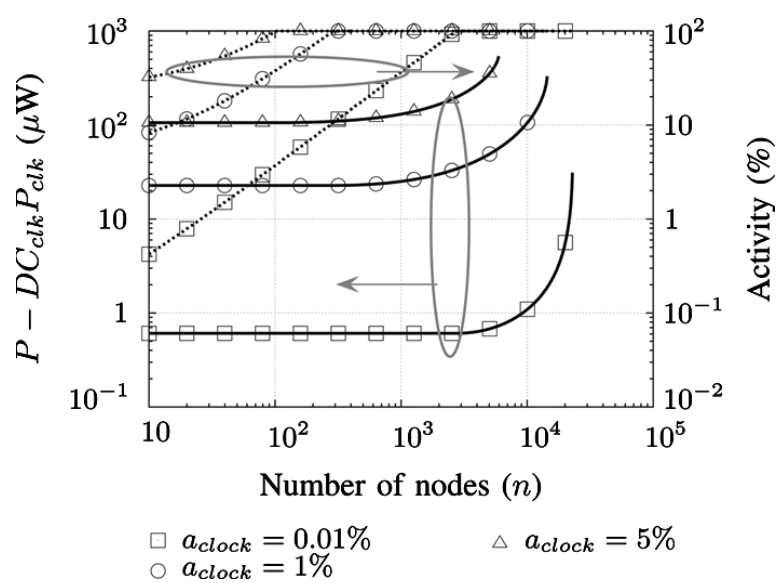

Fig. 3. Average power consumption of the radio section (wake-up radio and main radio) and network activity versus number of nodes; curves for three different values of clock accuracy are reported.

in each timeslot: for $a_{\text {clock }}=1 \%$ we obtain a maximum $n$ of around 14.400 and 3.600 nodes, respectively, for $\alpha=1$ and $\alpha=4$. Other sources of errors must be considered to give a more realistic prediction for the power $P$; in the next section, the loss in power introduced by nonidealities of the wake-up radio is calculated.

\section{Impact of Wake-Up Radio Nonidealities}

The operation of the wake-up radio is outlined here: the wake-up radio listens to the channel for a fixed time $T_{d}$ and decides if a useful signal is being received or not; if a wake-up request is detected, the main radio is turned on and it tries to synchronize to the incoming signal for the duration of the preamble $^{2}$; if synchronization succeeds, the payload is processed; otherwise the main radio is turned off, control is handed back to the wake-up radio and the whole operating cycle is repeated. We define a false alarm as the waking-up of the main radio when no useful signal has been received and a missed detection as a failure to wake up when a packet should have been received. If a node does not wake up to receive a packet, it will be retransmitted in a successive timeslot. The performance of the wake-up radio is then completely specified in terms of the probability that a particular decision will result in a false alarm $\left(\mathbb{P}_{\mathrm{fa}}\right)$, the probability that a particular decision will result in a missed detection $\left(\mathbb{P}_{\mathrm{md}}\right)$ and the time required to make these decisions $T_{d}$.

Taking these nonidealities into account, (4) and (5) have to be modified as

$$
\begin{aligned}
D C_{r x} & =\frac{N_{\mathrm{pr}}+N_{\mathrm{pl}}+n_{\mathrm{fa}} N_{\mathrm{pr}}}{D R}(P R+B R) \\
D C_{t x} & =\left(n_{\mathrm{md}}+1\right)\left(T_{d}+\frac{N_{\mathrm{pr}}+N_{\mathrm{pl}}}{D R}\right) P R
\end{aligned}
$$

where $n_{\mathrm{fa}}$ is the average number of false alarms issued per timeslot and $n_{\mathrm{md}}$ is the average number of missed detection per packet. Note that the transmitter must send a wake-up request

\footnotetext{
${ }^{2}$ For more details on synchronization, see Section IV-E.
} 


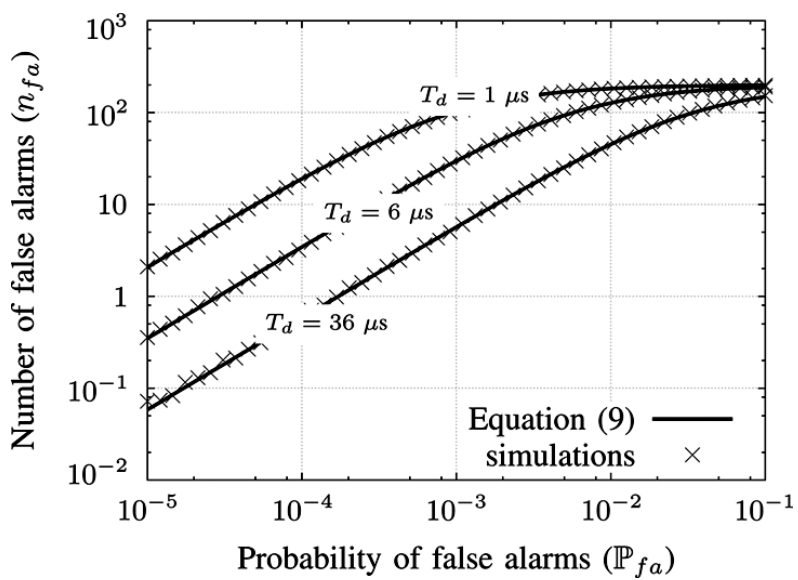

Fig. 4. Average number of false alarms per timeslots for different values of $T_{d}$ for the case $T_{\mathrm{wu}}=200 \mathrm{~ms}$ and $D R=100 \mathrm{kbps}$.

in an additional time $T_{d}$ before each packet in order to wake up the receiver.

The number of false alarms can be approximated as ${ }^{3}$

$$
n_{\mathrm{fa}} \approx \frac{\mathbb{P}_{\mathrm{fa}} T_{\mathrm{wu}}}{T_{d}+\frac{N_{\mathrm{pr}} \mathbb{P}_{\mathrm{fa}}}{D R}} .
$$

This approximated expression is in good accordance with simulations, as shown in Fig. 4, where (9) and the results of simulations are plotted for the case $T_{\mathrm{wu}}=200 \mathrm{~ms}$ and $D R=$ 100 kbps.

In order to find the requirements for $\mathbb{P}_{\mathrm{fa}}, \mathbb{P}_{\mathrm{md}}$ and $T_{d}$, we should know the relation between the power dissipated in the wake-up radio $P_{\mathrm{wu}}$ and the probability of errors. The knowledge of such a relation would enable the full optimization of total power $P$ : a lower probability of errors would decrease the duty-cycle of the main radio but the wake-up radio would then need to burn more power to achieve this. Moreover, a tradeoff exists for the choice of $T_{d}$ : a longer $T_{d}$ increases the transmission time but simultaneously reduces the probability of wake-up radio errors, as will be shown in Section V. Taking into account a possible implementation of the wake-up radio (see Section V), the following parameters have been chosen: $T_{d}=5.24 \mu \mathrm{s}$, $\mathbb{P}_{\mathrm{fa}}=2.6 \cdot 10^{-5}$ and $\mathbb{P}_{\mathrm{md}}=10^{-2}$, resulting in $n_{\mathrm{fa}} \approx 1$ and $n_{\text {md }} \approx 10^{-2}$.

In the previous analysis, the case in which a missed detection occurs during the reception of a synchronization beacon has been neglected. The node would simply skip that particular beacon and increase its $T_{\mathrm{wu}}$ until the reception of the next one. The effect on the power consumption is negligible and has been neglected in the above expressions.

\section{System Implementation and Performances}

The optimum parameters for the MAC scheme have been chosen using the guidelines described previously and are reported in Table II. To carry out the optimization, estimates have

\footnotetext{
${ }^{3}$ Equation (9) is not mathematically rigorous; it has been developed considering that $n_{\mathrm{fa}}$ is the product of the number of decisions to be taken in a timeslot by $\mathbb{P}_{\mathrm{fa}}$ and that the number of decisions depends on the difference between the duration of the timeslots and the time used for reception of the preamble caused by false alarms, i.e., $n_{\mathrm{fa}}=\mathbb{P}_{\mathrm{fa}}\left(T_{\mathrm{wu}}-n_{\mathrm{fa}} N_{\mathrm{pr}} / D R\right) / T_{d}$.
}

TABLE II

MAC PARAMETERS

\begin{tabular}{|l|l|l|l|}
\hline$D R$ & $100 \mathrm{kbps}$ & $B R$ & $0.2 \mathrm{~Hz}$ \\
\hline$T_{w u}$ & $200 \mathrm{~ms}$ & $T_{\text {guard }}$ & $50 \mathrm{~ms}$ \\
\hline$N_{p r}$ & 100 & $N_{p l}$ & 100 \\
\hline $\mathbb{P}_{\text {fa }}$ & $2.53 \cdot 10^{-3}$ & $\mathbb{P}_{m d}$ & $10^{-2}$ \\
\hline$a_{\text {clock }}$ & $1 \%$ & $\alpha$ & 4 \\
\hline
\end{tabular}

TABLE III

SYSTEM PERFORMANCES

\begin{tabular}{|l|r|r|r|}
\hline Subsystem & Peak power & Duty-cycle & Average power \\
\hline Main radio (Rx) & $2 \mathrm{~mW}$ & $0.065 \%$ & $1.3 \mu \mathrm{W}$ \\
\hline Main radio (Tx) & $2 \mathrm{~mW}$ & $0.003 \%$ & $0.1 \mu \mathrm{W}$ \\
\hline Wake-up radio & $500 \mu \mathrm{W}$ & $4.333 \%$ & $21.7 \mu \mathrm{W}$ \\
\hline Clock generator & $50 \mu \mathrm{W}$ & $100 \%$ & $50.0 \mu \mathrm{W}$ \\
\hline Total system & & & $73.0 \mu \mathrm{W}$ \\
\hline
\end{tabular}

been made of the peak power dissipated in the different subsystems, as was already briefly mentioned in Section III-B, and the accuracy of the clock generator has been fixed.

The specifications for the clock generator are based on the oscillator described in [13]. It consists of a low-voltage low-power fully integrated CMOS oscillator, which exploits the electron mobility in a MOS transistor as reference for the output frequency. There, it is shown that, with a power consumption less than $50 \mu \mathrm{W}$, an accuracy of the order of $1 \%$ can be reached over a wide temperature range, even when the effects of jitter and supply variations are taken into account. More accurate fully integrated oscillators, such as $L C$ oscillators, could also be employed, but this would be at the expense of increased power consumption [13]. The power consumption of the main radio in both receive and transmit modes is estimated to be in the order of a few milliwatts, taking into consideration the state of the art [14] and a transmitted power of $1 \mathrm{~mW}$ (see Section IV-B). However, the contribution of the main radio to the total average power is marginal and errors in the estimation of $P_{r x}$ and $P_{t x}$ can be largely tolerated. Wake-up radio performances and the required power consumption are described in Section V. With these assumptions for the peak power and with the duty-cycle factors derived from the MAC analysis, the average power consumption can be computed and is reported in Table III. The total average power is smaller than the allowed budget of $100 \mu \mathrm{W}$, demonstrating the feasibility of the system in WSN applications.

This analysis of the duty-cycled wake-up radio MAC protocol has shown the effect of clock inaccuracy on the power consumption of WSN node. It has also been proven that the overhead due to network synchronization can become the dominant fraction of power consumption when a fully integrated timing reference is adopted. Despite the low complexity of the proposed protocol, similar issues would arise when low-accuracy clock generators are used in more sophisticated protocols, such as T-MAC [15] or WiseMAC [7].

\section{E. Master Node}

A final remark should be made about the power consumption of the master node. The master node is equipped with the same hardware as the other nodes in the network: it is one of the nodes in the network which is elected as master during the setup 


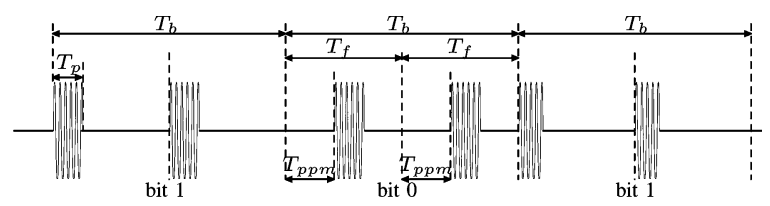

(a)

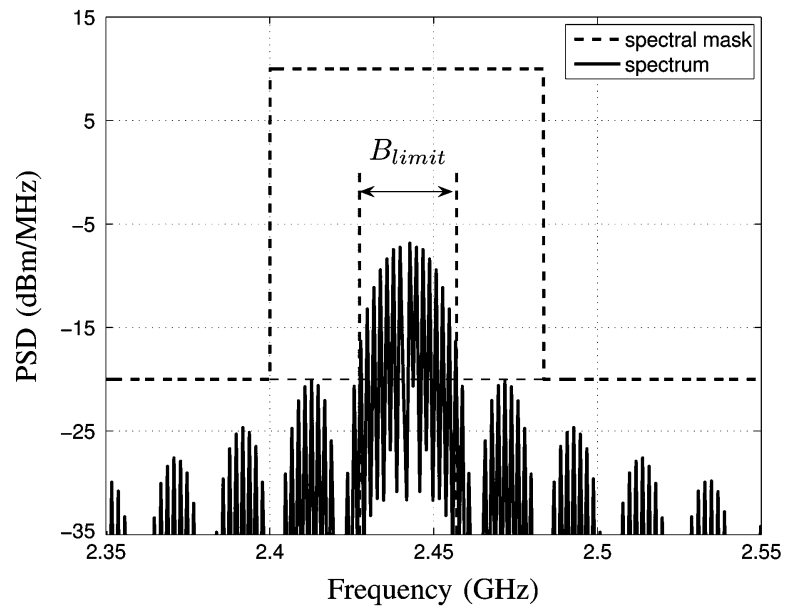

(b)

Fig. 5. (a) Example of IR signal. (b) Spectrum with parameters of Table IV.

phase of the network (not described in this work). Its power consumption differs slightly from that shown in the power budget of Table III, since it does not listen to the synchronization beacons, but sends them. This corresponds to a saving of $22 \mu \mathrm{W}$.

\section{IMPULSE RADIO MODULATION}

\section{A. Modulation and Band Allocation}

The RF modulation scheme must be chosen to relax the frequency accuracy requirements as much as possible. For narrowband modulation schemes (such as OOK, FSK, or QAM), the specifications on frequency accuracy are very strict, since the allowed frequency error at the receiver is directly proportional to the bandwidth of the RF signal. More suitable is an ultra-wideband (UWB) system employing a modulation scheme based on impulse radio (IR) [16]; the RF signal occupies a bandwidth of hundreds of megahertz to comply with radio regulations, and a fully integrated reference for this application can be easily built. However, it will be difficult to meet the power requirements at the receiver due to the inherent wideband nature of an UWB receiver. A better solution is the use of an Impulse Radio signal with a bandwidth smaller than that required in UWB systems but large enough to relax frequency accuracy constraints for full integration.

The preferred frequency band is the 2.4-GHz ISM band, which allows occupation of tens of megahertz at a frequency that is sufficiently high to enable the integration of the required passive components (e.g., inductors) on chip. Fig. 5 shows the simplified time representation of the adopted signal and its spectrum. An RF carrier is modulated by a pulse waveform with period $T_{f}$ and duty cycle $T_{p} / T_{f}$. The pulses are shaped as square waves with duration $T_{p}$ and pulse repetition frequency $P R F=1 / T_{f}$. Each bit is represented by a sequence of
$n_{p}=P R F / D R$ successive pulses, where $D R$ is the data rate, implementing the well-known repetition code. The bits are modulated using pulse position modulation (PPM). In each frame, i.e., in each slot of duration $T_{f}$ the pulse can be transmitted with different delays: the pulse can be positioned with zero delay (bit 0 ) or with delay $T_{\mathrm{ppm}}$ (bit 1 ), in case of binary modulation; more delays can be added to employ an $\mathrm{M}$-ary modulation. In the following, constraints affecting the modulation parameters choice are listed in order to find an optimal set of parameters.

\section{B. Transmitter Limits}

With an average transmitted power ${ }^{4} P_{\text {avg }}=1 \mathrm{~mW}$, the European ETSI 2.4-GHz ISM-band requirements are met if the transmission frequency resides in the interval $\left[f_{0}-\Delta f_{T X}, f_{0}+\Delta f_{T X}\right]$, where $f_{0}=2.44175 \mathrm{GHz}$ is the nominal transmitting frequency at the center of ISM band and

$$
\Delta f_{T X}=41.75 \mathrm{MHz}-\frac{B_{\text {limit }}\left(T_{p}\right)}{2}
$$

where the numerically computed $B_{\text {limit }}$ is the width of the spectrum of Fig. 5(b) at $-20 \mathrm{dBm} / \mathrm{MHz}$.

The complexity of transmitter circuitry depends on the signal Crest Factor, defined as $C F \triangleq P_{\text {peak }} / P_{\text {avg }}=T_{f} / T_{p}$ where $P_{\text {peak }}$ is the peak power. In order not to put excessive requirements on the transmitter, the crest factor has to be chosen less than $C F_{T X, \max }=10$. The following condition must hold:

$$
C F=\frac{T_{f}}{T_{p}} \leq \min \left\{C F_{T X, \max }, \frac{P_{\text {peak,limit }}}{P_{\text {avg }}}\right\}=10
$$

where $P_{\text {peak,limit }}$ is fixed by the regulations.

\section{Path Loss}

The signal power at the receiver antenna can be computed using the macroscopic model for the path loss [17]. The path loss is given by

$$
P L(d)=\left(\frac{4 \pi}{c} r_{0} f\right)^{2}\left(\frac{d}{r_{0}}\right)^{n_{P L}}
$$

where $c$ is light speed, $r_{0}=1 \mathrm{~m}$ is a reference distance, $d$ is the distance between transmitter and receiver antenna, $f$ is the signal frequency, and $n_{\mathrm{PL}}$ is the path loss coefficient. For indoor propagation, $n_{\mathrm{PL}}$ is usually in the range between 3 and 4. In the worst case $\left(n_{\mathrm{PL}}=4\right)$, with the parameters previously defined $(d=10, f=2.44 \mathrm{GHz})$ the path loss is $80 \mathrm{~dB}$. Taking into account an additional margin of $4 \mathrm{~dB}$, this corresponds to a line-of-sight path loss $\left(n_{\mathrm{PL}}=2\right)$ of $60 \mathrm{~dB}$ and to a margin of $24 \mathrm{~dB}$ for fading and obstructions effect. Since the transmitted power is $P_{\text {avg }}=1 \mathrm{~mW}$, the specification on receiver sensitivity is $-84 \mathrm{dBm}$.

\section{Receiver Limits}

To simplify the architecture, we assume the use of repetition coding and of a noncoherent receiver. In the following analysis,

${ }^{4}$ The power of the transmitted signal is limited by the power budget of the node; considering the power analysis carried in Section III and the expected efficiency of the transmitter, $P_{\text {avo }}=1 \mathrm{~mW}$ is a reasonable assumption. Note that the spectral mask requirements are still met if less power than $1 \mathrm{~mW}$ is emitted and the assumed value is a practical upper bound. 
the receiver is modeled as a matched filter followed by an ideal sampler [18]. With the adopted PPM scheme, the output of the matched filter is sampled twice per frame, i.e., in the position for bit 0 and for bit 1 , and the decision on the received bit is performed in the digital domain using a majority criterion: the bit is chosen according to the majority of the values of the demodulated pulses. For a fixed $P_{\mathrm{avg}}$ and $D R$, the implementation loss related to this simple decoding algorithm increases with the number of pulses per bit $n_{p}$; consequently, the following practical limit is posed:

$$
n_{p}=\frac{T_{b}}{T_{f}} \leq 25
$$

which corresponds to an implementation loss due to coding of $5.1 \mathrm{~dB}$ in receiver sensitivity for a bit error rate (BER) of $10^{-3}$. The loss can be overcome using a more complex coding scheme than repetition coding. If no coding gain is required but only the recovery of the loss, very simple schemes could be employed, which do not require complex digital hardware, but that is out of the scope of this work. Note that in terms of BER performances PPM is equivalent to the classical OOK modulation.

\section{E. Synchronization Requirements}

The receiver synchronizes itself in time and frequency to the incoming signal using the $N_{\mathrm{pr}}$ bits of the preamble. ${ }^{5}$ In case an imperfect synchronization is achieved, an implementation loss must be taken into account. It can be computed considering the equivalence between a matched filter and a correlation receiver and the need for two quadrature branches in a noncoherent receiver to tackle the phase difference between incoming signal at the antenna and local oscillator. Denoting with $x_{I}$ and $x_{Q}$ and with $y_{I}$ and $y_{Q}$ the in-phase and quadrature components, respectively, of the input and the output of the in-phase and quadrature correlation receivers, the detected energy of a single pulse after sampling can be expressed as 6

$$
\begin{aligned}
E_{p}\left(\Delta f_{\mathrm{syn}}, \Delta t_{\mathrm{syn}}\right)= & y_{I}^{2}+y_{Q}^{2} \\
= & \left(\int_{\Delta t_{\mathrm{syn}}}^{\Delta t_{\mathrm{syn}}+T_{p}} x_{I}(t) p(t) \mathrm{d} t\right)^{2} \\
& +\left(\int_{\Delta t_{\mathrm{syn}}}^{\Delta t_{\mathrm{syn}}+T_{p}} x_{Q}(t) p(t) \mathrm{d} t\right)^{2} \\
= & \frac{1-\cos \left[2 \pi \Delta f_{\mathrm{syn}}\left(T_{p}-\left|\Delta t_{\mathrm{syn}}\right|\right)\right]}{2\left(\pi \Delta f_{\mathrm{syn}}\right)^{2}}
\end{aligned}
$$

where $\Delta f_{\text {syn }}$ and $\Delta t_{\text {syn }}$ are, respectively, the error in frequency and timing between the actual value and the estimated one at

\footnotetext{
${ }^{5}$ The number of bits in the preamble is chosen to be sufficiently large to ease the implementation of the synchronization algorithm. At the same time, the choice $N_{\mathrm{pr}}=100$ does not affect sensibly the total power consumption (see Section III-D).

${ }^{6}$ Note that in the following we compute the detected energy of only one sampling, but in case of PPM two samplings per frame are needed. The adopted simplification is possible thanks to the equivalence in terms of energy and BER performance of PPM and OOK.
}

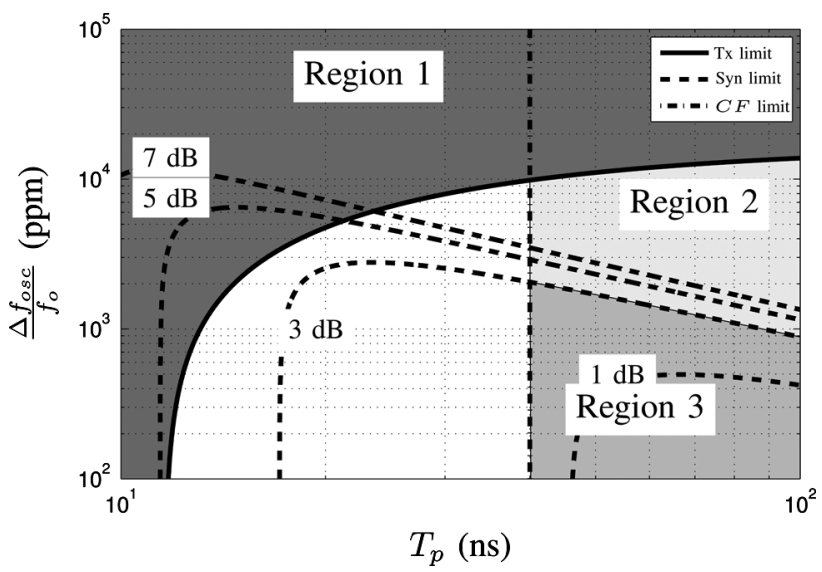

Fig. 6. Plot for the choice of $T_{p}$; different constraints are shown: Tx limit $\left(\Delta f_{\text {osc }} / f_{0} \leq \Delta f_{T X} / f_{0}\right)$, Syn limit $\left(\Delta f_{\text {osc }} / f_{0} \leq \Delta f_{\text {syn }} /\left(2 f_{0}\right)\right)$ for various $I L_{\mathrm{syn}}$ (shown on the curves) and CF limit (18).

the receiver, $p(t)$ is the pulse shape, defined for our modulation scheme as

$$
p(t)= \begin{cases}1, & \text { if } t \in\left[0, T_{p}\right] \\ 0, & \text { otherwise }\end{cases}
$$

and $x_{I}$ and $x_{Q}$ are given by

$$
\left\{\begin{array}{l}
x_{I}=p(t) \cos \left(2 \pi \Delta f_{\mathrm{syn}} t+\phi\right) \\
x_{Q}=p(t) \sin \left(2 \pi \Delta f_{\mathrm{syn}} t+\phi\right)
\end{array}\right.
$$

where $\phi$ is the constant phase difference between received signal and local oscillator. Since the performance of the matched filter receiver depends on the received energy, the implementation loss is

$$
\begin{aligned}
I L_{\mathrm{syn}} & =\frac{E_{p}(0,0)}{E_{p}\left(\Delta f_{\mathrm{syn}}, \Delta t_{\mathrm{syn}}\right)} \\
& =\frac{2\left(\pi \Delta f_{\mathrm{syn}} T_{p}\right)^{2}}{1-\cos \left[2 \pi \Delta f_{\mathrm{syn}}\left(T_{p}-\left|\Delta t_{\text {syn }}\right|\right)\right]} .
\end{aligned}
$$

\section{F. Optimization}

The choice of $T_{p}$ depends on $\Delta f_{\text {osc }}$, defined as the error of the local oscillator frequency with respect to the nominal frequency. All previous constraints are plotted in Fig. 6: $\Delta f_{\text {osc }} \leq \Delta f_{T X}$ [with reference to (10)] as a solid line; the $C F$ limit, i.e.,

$$
\frac{T_{f}}{T_{p}} \cdot \frac{T_{b}}{T_{f}} \leq 10 \cdot 25 \Rightarrow T_{p} \geq \frac{T_{b}}{250}=40 \mathrm{~ns}
$$

derived using (11) and (13), where $T_{b}$ is assumed to be $10 \mu \mathrm{s}(D R=100 \mathrm{kbps})$ in our application as a dashed-dotted line, and the synchronization limit as a dashed line. The synchronization limit is obtained from (17) for different values of $I L_{\mathrm{syn}}$ : the condition ${ }^{7} \Delta f_{\mathrm{osc}} \leq \Delta f_{\mathrm{syn}} / 2$ is plotted with the timing error of the synchronization algorithm fixed to $\left|\Delta t_{\mathrm{syn}}\right|=5 \mathrm{~ns}$. If the accuracy of the oscillator is enough for a given $I L_{\mathrm{syn}}$, no frequency estimation needs to be performed at the receiver. Since it is possible to trade off timing error

\footnotetext{
${ }^{7}$ The factor 2 derives from the presence of frequency errors both in transmitter
} and receiver. 
TABLE IV

MODULATION PARAMETERS

\begin{tabular}{|l|l|l|l|l|l|}
\hline \multicolumn{2}{|l|}{ Data rate $(D R)$} & $100 \mathrm{kbps}$ & $P_{\text {avg }}$ & $1 \mathrm{~mW}$ \\
\hline \multicolumn{2}{|l|}{$-3 \mathrm{~dB}$ bandwidth } & $17.7 \mathrm{MHz}$ & $C F$ & 9.52 \\
\hline IR parameters & $T_{f}$ & $476 \mathrm{~ns}$ & Synchro- & $I L_{\text {syn }}$ & $3 \mathrm{~dB}$ \\
& $T_{p p m}$ & $238 \mathrm{~ns}$ & nization & $\Delta f_{\text {syn }}$ & $8.4 \mathrm{MHz}$ \\
& $T_{p}$ & $50 \mathrm{~ns}$ & parameters & $\Delta t_{\text {syn }}$ & $5 \mathrm{~ns}$ \\
\hline
\end{tabular}

$\left(\Delta t_{\mathrm{syn}}\right)$ for frequency error $\left(\Delta f_{\mathrm{syn}}\right)$ in (17), a small timing error $(5 \mathrm{~ns})$ has been chosen. This is advantageous in terms of hardware as it is more difficult to tune the frequency of the receiver than the timing.

From Fig. 6, a good choice for $T_{p}$ can be found using frequency accuracy considerations. Referring to an $I L_{\mathrm{syn}}$ of $3 \mathrm{~dB}$, it is possible to distinguish different regions. In region 1, i.e., all of the points above the transmission limit, the transmitter will not respect the spectral mask. Points in region 2 respect the transmission limit, but some frequency synchronization at the receiver is needed because the frequency accuracy is not good enough. Region 3, i.e., the points under the transmission limit and under the curve of $I L_{\mathrm{syn}}=3 \mathrm{~dB}$, contains points for which no frequency synchronization is required at the receiver because the frequency accuracy provided by the local oscillator is enough to maintain $I L_{\mathrm{syn}}$ below $3 \mathrm{~dB}$. The optimal point is strictly related to the available oscillator frequency accuracy. For example with an accuracy of $0.2 \%$, it can be seen that a choice of $T_{p}=40 \mathrm{~ns}$ corresponds to a point in the optimal region 3. However, if an accurate frequency reference, e.g., a crystal oscillator, is not available, it will be very challenging to achieve accuracies below $1 \%$ [13]. At the $1 \%$ level of accuracy, frequency synchronization must be performed for any value of $T_{p}$, in order to keep the implementation loss due to the synchronization system less than $3 \mathrm{~dB}$. A choice of $T_{p}=50 \mathrm{~ns}$ has been made, leading to an allowed absolute timing and frequency error equal to $5 \mathrm{~ns}$ and $8.44 \mathrm{MHz}$ respectively.

The frame period $T_{f}$ is chosen according to different requirements: (11), (13), and $P R F<2.5 \mathrm{Mpps}$ (to avoid a very fast baseband). Thus, $T_{f}=476 \mathrm{~ns}$ is employed and, consequently, $P R F=2.1 \mathrm{Mpps}, n_{p}=21$ and $C F=9.52$. The resulting modulation parameters are reported in Table IV.

\section{WAKE-UP RADIO}

\section{A. Architecture and Performance}

As the wake-up radio is used instead of the main radio during channel idle listening, its power consumption must be much lower than that of the main radio in order to make the substitution advantageous. This is possible thanks to the wake-up radio's relaxed specifications, since its functionality is limited to the recognition of an incoming IR wake-up request. The required specifications for the wake-up radio obtained from a system level analysis have been previously discussed and are summarized in Table V.

In the literature, the wake-up radio is often referred to as an ultra-low-power radio which is always active and causes the whole radio to react to useful incoming useful signals [19]. With this approach, the wake-up radio's power consumption
TABLE V

WAKE-UP RADIO REQUIREMENTS

\begin{tabular}{|l|l|}
\hline Power consumption $\left(P_{w u}\right)$ & $500 \mu \mathrm{W}$ \\
\hline Probability of false alarm $\left(\mathbb{P}_{f a}\right)$ & $2.6 \cdot 10^{-5}$ \\
\hline Probability of missed detection $\left(\mathbb{P}_{m d}\right)$ & $10^{-2}$ \\
\hline Wake-up decision time $\left(T_{d}\right)$ & $5.24 \mu \mathrm{s}$ \\
\hline
\end{tabular}

must be less than the total available power, i.e., $100 \mu \mathrm{W}$ in the present case. In order to meet such a low power consumption, previous solutions have used very simple architectures, avoiding local oscillator and complex down-conversion architectures [20] or employing a free-running oscillator periodically calibrated with an external precise frequency [19]. In the latter, power consumption is reduced by avoiding power-hungry PLLs, but a precise frequency reference, which cannot be integrated, is still required. On the other hand, if down-conversion architectures, like super-heterodyne, low-IF, or direct-conversion architectures, are not used, a narrow RF channel filter will be required, which can only be implemented by employing high- $Q$ external RF filters, such as BAW filters or MEMS resonators [8], [9]. Narrow channel filtering can not be avoided if a good sensitivity at ultra-low-power consumption is required. If the bandwidth is not limited, indeed, the signal may be buried under the noise picked up by the antenna and added by the radio's front-end; the latter can be reduced only at the expense of higher power dissipation.

Our solution overcomes these limits at two different levels. At the network level, the adoption of a TDMA scheme allows duty-cycling of the wake-up radio, relaxing in this way the requirements on its instantaneous power dissipation, which can be as high as $500 \mu \mathrm{W}$, as proven in Section III. The increased power budget available for the wake-up radio will reduce the noise added by the circuitry and increase the sensitivity. At the physical level, the use of IR is beneficial for the wake-up functionality. Since the peak power of the pulses is higher than the average power of the incoming signal, it is easier for the wake-up radio to discriminate in the amplitude domain a useful signal from noise. Thus, on the one hand, the higher available power allows the design of circuits with lower noise and, on the other hand, the system is more immune to the effect of noise thanks to IR modulation. In this way, the wake-up radio does not need a narrowband filtering and it can be implemented at low cost in standard integrated circuit technology.

A possible implementation of a wake-up radio and the representation of signals for each block are shown in Fig. 7(a) and (b), respectively. When the signal of Fig. 5(a) is present at the antenna and an appropriate threshold is chosen, the output of the envelope detector consists of a noisy train of pulses down-converted to the baseband. A comparator is used to discriminate the useful signal from the noise and in case of a clean signal its output is a square wave with the same shape of the envelope of the IR signal. A pulsewidth detector verifies that the pulses are longer than a certain pulse length threshold in order to prevent the system to give false alarms in case of short and high noise peaks.

As for a standard radio, it is possible to define the sensitivity of a wake-up radio. The sensitivity is defined as the minimum 


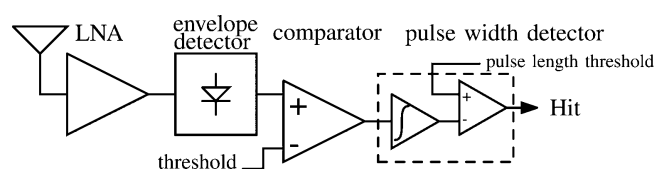

(a)

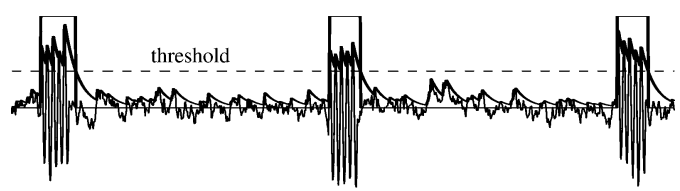

(b)

Fig. 7. (a) Wake-up radio architecture and (b) example-related waveforms: the outputs of LNA, envelope detector, and comparator are shown.

signal level that the system can detect with acceptable performances. The requirement on the sensitivity has been fixed to $-84 \mathrm{dBm}$ in Section IV-C. The acceptable performances have been defined in Section III-C in terms of $\mathbb{P}_{\mathrm{fa}}, \mathbb{P}_{\mathrm{md}}$ and $T_{d}$. For the architecture in Fig. 7(a), the sensitivity is given by [21]

$$
S=k T \cdot N F \cdot B_{n} \cdot S N R
$$

where $k$ is the Boltzmann constant, $T$ is the absolute temperature, $B_{n}$ is the receiver noise bandwidth, $N F$ is the noise figure of the LNA, and $S N R$ is the minimum signal-to-noise ratio at the input of the envelope detector, which is needed to achieve the required $\mathbb{P}_{\text {fa }}$ and $\mathbb{P}_{\text {md }}$. Once the minimum $S N R$ is known, from (19) it is possible to find $N F$, which allows to estimate the power consumption of the wake-up radio. The minimum required $S N R$ will be computed in the following.

We define the missed pulse probability $\left(p_{\mathrm{mp}}\right)$ as the probability to miss a single incoming pulse and the false pulse probability $\left(p_{\mathrm{fp}}\right)$ as the probability to detect a noise peak as an incoming pulse. The relation between $p_{\mathrm{fp}}$ and $p_{\mathrm{mp}}$ and the network-level parameters $\mathbb{P}_{\text {fa }}$ and $\mathbb{P}_{\mathrm{md}}$ is easy to find in the case when $m$ pulses are transmitted to wake up the receiver. We suppose that the wake-up radio of Fig. 7(a) checks the presence of a pulse inside each frame of duration $T_{f}$. The digital output of the wake-up radio (hit) is high when a pulse is detected, and, after each frame, the system stores the value of the digital output. After the observation of $m$ frames periods, the number of hits, i.e., the number of times a pulse was detected in $m$ frames, is counted. If that number is larger than a fixed value $n_{\mathrm{th}}$, a wake-up call to the main radio is issued. Otherwise the count is reset and the procedure is started again. In this scenario $\mathbb{P}_{\text {fa }}$ and $\mathbb{P}_{\text {md }}$ are given by the following equations:

$$
\begin{aligned}
\mathbb{P}_{\mathrm{fa}} & =\sum_{k=n_{\text {th }}+1}^{m}\left(\begin{array}{c}
m \\
k
\end{array}\right) p_{\mathrm{fp}}^{k}\left(1-p_{\mathrm{fp}}\right)^{m-k} \\
\mathbb{P}_{\mathrm{md}} & =\sum_{k=0}^{n_{\mathrm{th}}}\left(\begin{array}{c}
m \\
k
\end{array}\right)\left(1-p_{\mathrm{mp}}\right)^{k} p_{\mathrm{mp}}^{m-k} .
\end{aligned}
$$

Equations (20) and (21) are plotted in Fig. 8 in the case of $m=11$, which corresponds to $T_{d}=5.24 \mu$ s (see Table IV).

Matlab simulations were performed to find the relation between $p_{\mathrm{fp}}, p_{\mathrm{mp}}$ and $S N R$ for the architecture of Fig. 7(a). They can be computed by evaluating the probability of a hit in a frame

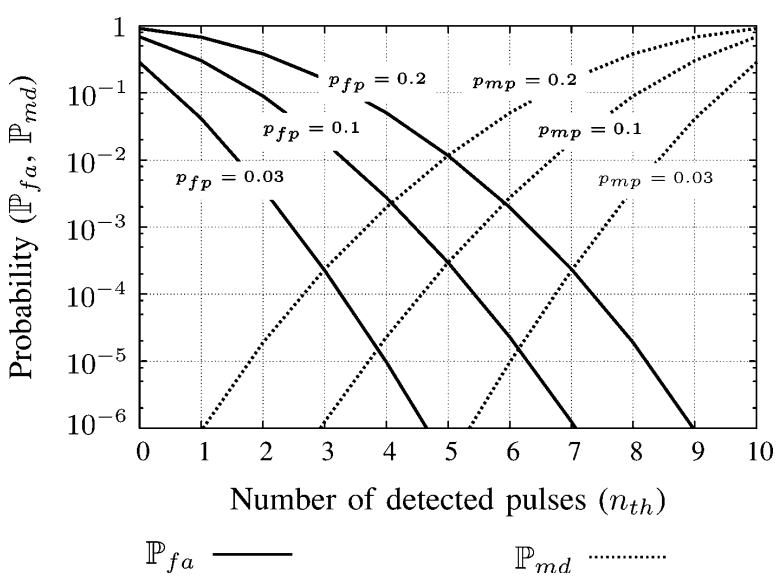

Fig. 8. Probability of false alarm $\mathbb{P}_{\mathrm{fa}}$ and probability of missed detection $\mathbb{P}_{\text {md }}$ versus number of detected pulses $n_{\mathrm{th}}$; curves are plotted for fixed values of $m=11$ and the values of $p_{\mathrm{fp}}$ and $p_{\mathrm{mp}}$ reported in the figure.

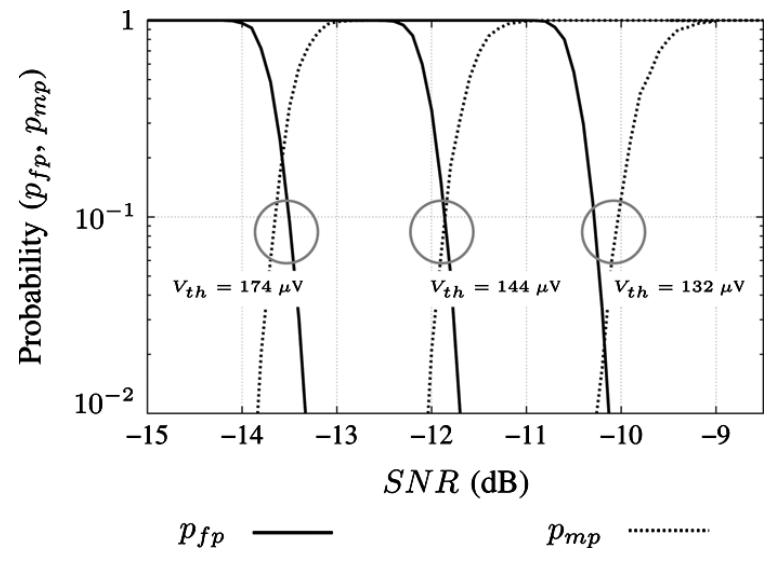

Fig. 9. Probability of false pulse and probability of missed pulse versus signal to noise ratio $S N R$; curves are plotted for different values of $V_{\mathrm{th}}$.

where a pulse is present and the probability of a hit in a frame with no pulses but only noise. An IR waveform with a signal power equal to the sensitivity of the main radio $(S=-84 \mathrm{dBm})$ at $2.44 \mathrm{GHz}$ and with a $C F=9.52\left(T_{f}=476 \mathrm{~ns}, T_{p}=50 \mathrm{~ns}\right)$ was fed to the antenna. An AWGN channel was assumed and the $S N R$ at the input of the envelope detector was swept. ${ }^{8}$ The threshold of the pulsewidth detector is set to $45 \mathrm{~ns}$, the envelope detector has an exponential discharge behavior with time constant of $10 \mathrm{~ns}$ and the comparator has a bandwidth of $40 \mathrm{MHz}$. The threshold of the comparator $V_{\text {th }}$ has a dramatic impact on the performance of the wake-up radio: it should be high enough to minimize $p_{\mathrm{fp}}$ and low enough to minimize $p_{\mathrm{mp}}$. To determine the effect of $V_{\mathrm{th}}$ on the wake-up radio performance, $p_{\mathrm{fp}}$ and $p_{\mathrm{mp}}$ were computed with different threshold values and results are summarized in Fig. 9.

An optimum choice for $n_{\mathrm{th}}$ and $V_{\mathrm{th}}$ exists, which minimizes the $S N R$ required to achieve $\mathbb{P}_{\mathrm{fa}}$ and $\mathbb{P}_{\mathrm{md}}$ in Table $\mathrm{V}$. From inspection of Fig. 8, it may be seen that, with $n_{\mathrm{th}}=6$,

${ }^{8}$ Though the analysis was carried on with an AWGN channel, it must be noted that a margin for fading and obstruction has been considered in Section IV-C Propagation through a multipath channel only affects the energy received for each pulse, since the delay between successive pulses is much higher than multipath delay expected by typical channel models. The effect of interferers is treated in Section V-B. 
$p_{\mathrm{fp}}=10^{-1}$ and $p_{\mathrm{mp}}=10^{-1}$ satisfy the network requirements on $\mathbb{P}_{\mathrm{fa}}$ and $\mathbb{P}_{\mathrm{md}}$. Fig. 9 shows that, with the choice $V_{\mathrm{th}}=144 \mu \mathrm{V}$, an $S N R$ of $-11.8 \mathrm{~dB}$ is required to achieve both the condition $p_{\mathrm{fp}}<10^{-1}$ and $p_{\mathrm{mp}}<10^{-1}$. It can be proven that the choice $n_{\mathrm{th}}=6$ and $V_{\mathrm{th}}=144 \mu \mathrm{V}$ is a good approximation of the minimum $S N R$. If $V_{\mathrm{th}}>144 \mu \mathrm{V}$ is chosen, there are no values of $S N R$ and $n_{\text {th }}$ for which both the conditions on $\mathbb{P}_{\mathrm{fa}}$ and $\mathbb{P}_{\mathrm{md}}$ are met. Finally, if $V_{\mathrm{th}}<144 \mu \mathrm{V}$, a range of values for $n_{\text {th }}$ exists for which the system over-performs the requirements in terms of $\mathbb{P}_{\mathrm{fa}}$ and $\mathbb{P}_{\mathrm{md}}$. However, in that case a higher $S N R$ is required, with requires more power consumption in the front-end.

The optimum $S N R$ is dependent on the decision time $T_{d}$. A lower $S N R$ can be obtained by increasing the number of pulses $m$ and appropriately adjusting the threshold $n_{\mathrm{th}}$ : in that case, higher $p_{\mathrm{fp}}$ and $p_{\mathrm{mp}}$ can be allowed to achieve a fixed $\mathbb{P}_{\mathrm{fa}}$ and $\mathbb{P}_{\text {md }}$ (see Fig. 8). On the other hand, higher $p_{\mathrm{fp}}$ and $p_{\mathrm{mp}}$ allows a lower $S N R$ by increasing the threshold $V_{\text {th }}$ (see Fig. 9) and, consequently, a higher $N F$ for the LNA can be accepted while maintaining the same performances. Even though a higher value of $m$ decreases the power of the wake-up radio, it results in a longer $T_{d}$, which, as was already discussed in Section III-C, increases the power spent in the transmitter. To carry out the optimization on $T_{d}$, the relation between wake-up radio power and $N F$ should be known. As a simple topology-independent relation could not be found, a reasonable value of $T_{d}=5.24 \mu \mathrm{s}$ has been chosen.

\section{B. Effect of Interferers}

Interferers can erroneously trigger the wake-up radio, thereby increasing the power consumption of the whole system. Due to the absence of a narrowband filter in the wake-up radio, any interferer in the antenna's bandwidth causes a false alarm with a probability dependent on the power of the interferer, exactly in the same way as the probability of missed detection is dependent on the power of the received signal. We take into consideration an interferer that has equal probability of generating a false alarm as the minimum signal detectable by the wake-up radio. This corresponds, for a nonpulse-based interferer to a power equal to

$$
P_{\mathrm{int}}=S \cdot C F=-74 \mathrm{dBm}
$$

where $S$ is the receiver sensitivity and $C F$ is the crest factor defined in Section IV-B. It is clear that a gain equal to the crest factor is achieved in interference immunity thanks to the adoption of a pulse-based modulation scheme. In order to estimate the number of false alarms generated by interferers, we need to define an interferer scenario and the rate of appearance of such signals, which is not defined by spectrum regulations. As an example of a typical scenario, we consider the case of Bluetooth interferers caused by communication between a Bluetooth device, such as a mobile phone or a computer, and a Bluetooth headset. This is not sufficient to draw extremely general conclusions, but it allows us to sketch the activity of the main radio in a typical scenario. If any of the two Bluetooth devices is close enough to the node under consideration, a false alarm is issued when the Bluetooth device uses the channel. Bidirectional audio transmission between the headset and the other device typically generates traffic consisting of periodic repetition of bursts of two packets $^{9}$ (called SCO packets in the Bluetooth standard) with period $^{10}$ equal to $1.25,2.5$, or $3.75 \mathrm{~ms}$ [22]. In this scenario, the number of false alarms for each timeslot is, respectively, 160 , 80 , and 40 for the different repetition rates and the increase in system power consumption, due to increase of the duty-cycle of the main radio, is, respectively, 64,32 , and $16 \mu \mathrm{W}$.

In order to save energy in such a scenario, several strategies can be adopted. One strategy consists, for example, in limiting the number of wake-up requests for each timeslot. When, in a certain timeslot, the main radio is woken up by interferers more times than a certain maximum (defined as a parameter of the MAC protocol), it will not wake up anymore. Data transmission could then be delayed to other timeslots, during which the channel is not used by interferers. This strategy results in additional latency, which, however, can be tolerated in most applications. An alternative strategy consists in increasing the threshold of the comparator until the interferers can not trigger the wake-up radio. Increasing the threshold reduces the sensitivity and, consequently, the range between transmitter and receiver, i.e., $d$ in (12). This strategy is particularly suitable for dense WSNs, in which information can be routed through multiple hops, in order to cover a longer distance with a sequence of shorter hops. The spatial redundancy of nodes typical of WSN can then be exploited to give robustness over interferers while maintaining low power consumption per node. Both strategies increase the packet latency and/or the packet rate, but this can always be accepted in low QoS applications. On the other hand, the system presented in this work could also implement applications with more stringent requirements on QoS. In that case, power consumption can be traded for QoS, by allowing interferers to wake up the main radio and, consequently, giving up the power saving gained by the introduction of the duty-cycled wake-up radio.

\section{Notes on Implementation}

Using the $S N R$ found in the previous section and (19), the $N F$ of the wake-up radio front-end can be derived. It is then possible to evaluate the feasibility of the proposed architecture in terms of power consumption. With no narrowband filtering at the antenna, the noise bandwidth of the system is assumed to be wider than $100 \mathrm{MHz}$. For 100-MHz noise bandwidth and $-11.8 \mathrm{~dB} S N R$, an $N F$ of $21.6 \mathrm{~dB}$ is needed for a sensitivity of $-84 \mathrm{dBm}$. The envelope detector requires a nonlinear device such as a diode. In this case, a minimum allowable signal of $60 \mathrm{mV}_{\text {peak }}$ amplitude at the envelope detector's input is required. Thus, the voltage gain of the block preceding the envelope detector should have a voltage gain of $60 \mathrm{~dB}$ when an antenna impedance of $50 \Omega$ and the parameters of Table IV are used. Accordingly, the threshold for the comparator should be scaled to $144 \mathrm{mV}$. It seems feasible to reach the specifications on gain and noise figure with $500 \mu \mathrm{W}$ power consumption [8].

\footnotetext{
${ }^{9}$ Each packet has a duration of $366 \mu$ s and the delay between the first and secon packet in the burst is $625 \mu \mathrm{s}$

${ }^{10}$ The period is adapted to the QoS required for the audio link.
} 


\section{CONCLUSION}

It has been shown how low power consumption and full integration can be achieved in a node for WSN if an ad hoc MAC and modulation scheme are employed. We have introduced a MAC scheme based on a TDMA approach and on the use of a wake-up radio duty-cycled by an on-board clock generator. The accuracy of the clock generator has a significant impact on system performances, in terms of average power consumption and maximum number of nodes allowed in the network. For moderate and low clock accuracy, the energy overhead due to network synchronization becomes a dominant fraction of the power consumption. It has been proven that each node in the WSN can be expected to dissipate a total average power of less than $100 \mu \mathrm{W}$ if the inaccuracy of the clock generator is in the order of $1 \%$. As such level of accuracy is compatible with the performance of state-of-the-art fully integrated frequency references, no crystal oscillators or other external references are needed in the implementation of the proposed scheme. The issue of integration has also been solved with regards to the RF communication, introducing an Impulse Radio modulation scheme in the 2.4-GHz ISM band. The modulation parameters have been optimized to relax the required frequency accuracy to the order of $1 \%$, by taking into account the constraints due to spectral mask and crest factor for the transmitter and to the synchronization for the receiver. Finally, the performance of the wake-up radio has been analyzed with respect to the effect on the MAC scheme and its feasibility for the IR modulation without any external components (e.g., BAW filters and MEMS) has been proven. The impact of interferers on wake-up radio performances and, consequently, on the power consumption of the total system has been analyzed, and the tradeoff between power consumption and QoS in presence of interferers has been briefly discussed.

\section{REFERENCES}

[1] , W. Weber, J. M. Rabaey, and E. Aarts, Eds., Ambient Intelligence. New York: Springer, 2005.

[2] I. Akyildiz, W. Su, Y. Sankarasubramaniam, and E. Cayirci, "A survey on sensor networks," IEEE Commun. Mag., vol. 40, no. 8, pp. 102-114, Aug. 2002.

[3] B. W. Cook, S. Lanzisera, and K. S. J. Pister, "SoC issues for RF smart dust," Proc. IEEE, vol. 94, no. 6, pp. 1177-1196, Jun. 2006.

[4] S. Chalasani and J. Conrad, "A survey of energy harvesting sources for embedded systems," in Proc. Southeastcon, Apr. 2008, pp. 442-447.

[5] J. Ammer, F. Burghardt, E. Lin, B. Otis, R. Shah, M. Sheets, and J. M. Rabaey, "Ultra low-power integrated wireless nodes for sensor and actuator networks," in Ambient Intelligence, W. Weber, J. M. Rabaey, and E. Aarts, Eds. New York: Springer, 2005.

[6] J. M. Rabaey, J. Ammer, T. Karalar, L. Suetfei, B. Otis, M. Sheets, and T. Tuan, "PicoRadios for wireless sensor networks: The next challenge in ultra-low power design," in ISSCC Dig. Tech. Papers, Feb. 2002, vol. 1, pp. 200-201.

[7] C. C. Enz, A. El-Hoiydi, J.-D. Decotignie, and V. Peiris, "Wisenet: An ultralow-power wireless sensor network solution," Computer, vol. 37, no. 8, pp. 62-70, Aug. 2004.

[8] D. C. Daly and A. P. Chandrakasan, "An energy-efficient OOK transceiver for wireless sensor networks," IEEE J. Solid-State Circuits, vol. 42, no. 5, pp. 1003-1011, May 2007.

[9] B. W. Otis, Y. H. Chee, R. Lu, N. M. Pletcher, and J. M. Rabaey, "An ultra-low power MEMS-based two-channel transceiver for wireless sensor networks," in VLSI Circuits, Dig. Tech. Papers, Jun. 2004.
[10] K. Romer and F. Mattern, "The design space of wireless sensor networks," IEEE Wireless Commun., vol. 11, no. 6, pp. 54-61, Dec. 2004.

[11] J. Al-Karaki and A. Kamal, "Routing techniques in wireless sensor networks: A survey," IEEE Wireless Commun., vol. 11, no. 6, pp. 6-28, Dec. 2004

[12] C. C. Enz, N. Scolari, and U. Yodprasit, "Ultra low-power radio design for wireless sensor networks," in Proc. IEEE Int. Workshop Radio-Frequency Integration Technol., Nov. 2005, pp. 1-17.

[13] F. Sebastiano, L. Breems, K. Makinwa, S. Drago, D. Leenaerts, and B. Nauta, "A low-voltage mobility-based frequency reference for crystalless ULP radios," in Proc. ESSCIRC, Sep. 2008, pp. 306-309.

[14] B. W. Cook, A. Berny, A. Molnar, L. Steven, and K. S. J. Pister, "Lowpower 2.4-GHz transceiver with passive RX front-end and 400-mV supply," IEEE J. Solid-State Circuits, vol. 41, no. 12, pp. 2757-2766, Dec. 2006.

[15] T. van Dam and K. Langendoen, "An adaptive energy-efficient MAC protocol for wireless sensor networks," in Proc. 1st ACM Conf. Embedded Networked Sensor Systems (SenSys), Nov. 2003, pp. 171-180.

[16] J. Ryckaert, C. Desset, A. Fort, M. Badaroglu, V. De Heyn, P. Wambacq, G. Van der Plas, S. Donnay, B. Van Poucke, and B. Gyselinckx, "Ultra-wide-band transmitter for low-power wireless body area networks: Design and evaluation," IEEE Trans. Circuits Syst. I, Reg. Papers, vol. 52, no. 12, pp. 2515-2525, Dec. 2005.

[17] H. Hashemi, "The indoor radio propagation channel," Proc. IEEE, vol. 81, no. 7, pp. 943-968, Jul. 1993.

[18] L. W. Couch, II, Digital and Analog Communication Systems. Upper Saddle River, NJ: Prentice-Hall PTR, 1992.

[19] N. M. Pletcher and S. Gambini, "A $2 \mathrm{GHz} 52 \mu \mathrm{W}$ wake-up receiver with $-72 \mathrm{dBm}$ sensitivity using uncertain-IF architecture," in IEEE ISSCC Dig. Tech. Papers, Feb. 2008, pp. 524-525.

[20] N. Pletcher, S. Gambini, and J. Rabaey, "A $65 \mu \mathrm{W}, 1.9 \mathrm{GHz}$ RF to digital baseband wakeup receiver for wireless sensor nodes," in Proc. Custom Integrated Circuits Conf., Sep. 2007, pp. 539-542.

[21] B. Razavi, RF Microelectronics. Upper Saddle River, NJ: PrenticeHall, 1998.

[22] Specifications of the Bluetooth System, , Jul. 2007, Std., Rev. 2.1.

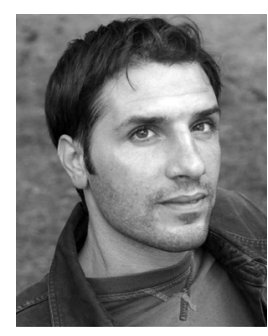

Salvatore Drago received the M.Sc. degree (cum laude) in electrical engineering from the University of Catania, Catania, Italy, in 2003. He is currently working toward the Ph.D. degree at the University of Twente, Enschede, The Netherlands.

From 2004 to 2006, he was with Synapto s.r.l., Catania, where he worked on EM modeling of embedded passives and interconnections in PCBs. In 2006 he joined NXP Semiconductors Research, Eindhoven, The Netherlands, as a Marie Curie Fellow. His research interests include ultra-low-power radios and RF integrated circuit design.

Mr. Drago was a corecipient of the 2008 ISCAS Best Student Paper Award.

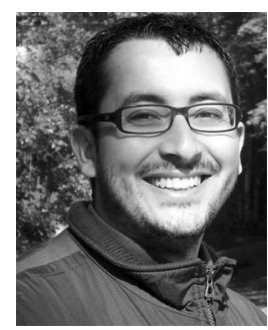

Fabio Sebastiano was born in Teramo, Italy, in 1981. He received the B.Sc. (cum laude) and M.Sc. (cum laude) degrees in electrical engineering from the University of Pisa, Pisa, Italy, in 2003 and 2005, respectively, and the Diploma di Licenza from Scuola Superiore Sant'Anna, Pisa, Italy, in 2006. He is currently working toward the Ph.D. degree at Delft University of Technology, Delft, The Netherlands.

In 2006, he joined NXP Semiconductors Research, Eindhoven, The Netherlands, as a Marie Curie Fellow. His main research interests are ultra-low power radios for wireless sensor networks and fully integrated crystal-less frequency references.

Mr. Sebastiano was a corecipient of the 2008 ISCAS Best Student Paper Award. 


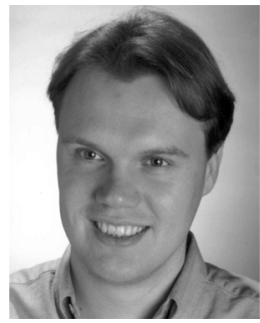

Lucien J. Breems (S'97-M'00-SM'07) received the M.Sc. degree and the Ph.D. degree in electrical engineering from the Delft University of Technology, Delft, The Netherlands, in 1996 and 2001, respectively.

From 2000 to 2006, he was with Philips Research Laboratories, Eindhoven, The Netherlands, and in 2007 he joined NXP Semiconductors Research, Eindhoven. He is author of the book Continuous-Time Sigma-Delta Modulation for A/D Conversion in Radio Receivers (Kluwer, 2001), and his research interests include sigma-delta modulators and mixed-signal circuit design. He has been an Invited Lecturer with Delft University of Technology, Delft, The Netherlands, since 2008.

Dr. Breems is a member of the technical program committee of the IEEE Symposium on VLSI Circuits and he has served as an Associate Editor for the IEEE TRANSACTIONS ON CIRCUITS AND SYSTEMS-II: EXPRESS BRIEFS (2008-2009). He received the Jan van Vessem Best Paper Award at the 2001 IEEE International Solid-State Circuits Conference.

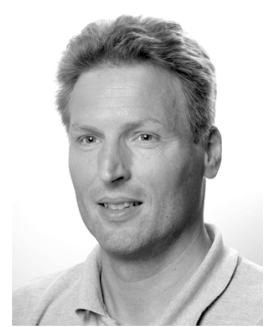

Domine M. W. Leenaerts (M'94-SM'96-F'05) received the $\mathrm{Ph} . \mathrm{D}$. degree in electrical engineering from Eindhoven University of Technology, Eindhoven, The Netherlands, in 1992.

From 1992 to 1999, he was with Eindhoven University of Technology as an Associate Professor with the Micro-electronic Circuit Design group. In 1995, he was a Visiting Scholar with the Department of Electrical Engineering and Computer Science, University of California, Berkeley. In 1997, he was an Invited Professor with Ecole Polytechnique Federale de Lausanne, Lausanne, Switzerland. From 1999 to 2006, he was a Principal Scientist with Philips Research Laboratories, Eindhoven, where he was involved in RF integrated transceiver design. In 2006, he joined NXP Semiconductors Research, Eindhoven, as a Senior Principal Scientist. He has published over 150 papers in scientific and technical journals and conference proceedings and he holds several U.S. patents. He has coauthored several books, including Circuit Design for RF Transceivers (Kluwer, 2001).

Dr. Leenaerts served as an IEEE Distinguished Lecturer during 2001-2003 and served as an Associate Editor of the IEEE TRANSACTIONS ON CIRCUITS AND SYSTEMS-I: REGULAR PAPERS (2002-2004) and since 2007 has been a Associate Editor of the IEEE Journal of Solid-State CIRCUITS. During 2005-2008, he was an IEEE Circuits and Systems Society Member representative in the IEEE Solid-State Circuits Society Administrative Committee, on which he is now an elected member. He serves currently on the Technical Program Committee of the European Solid-State Circuits Conference, the IEEE Radio Frequency Integrated Circuits (RFIC), and the IEEE International Solid-State Circuits Conference (ISSCC).

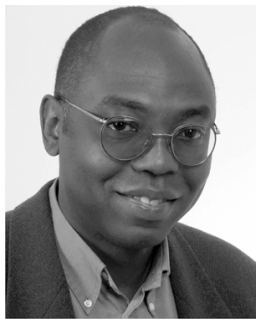

Kofi A. A. Makinwa (M'97-SM'05) received the B.Sc. and M.Sc. degrees from Obafemi Awolowo University, Nigeria, in 1985 and 1988, respectively, the M.E.E. degree from the Philips International Institute, The Netherlands, in 1989, and the Ph.D. degree from Delft University of Technology, Delft, The Netherlands, in 2004.

From 1989 to 1999, he was a Research Scientist with Philips Research Laboratories, where he worked on interactive displays and on front-ends for optical and magnetic recording systems. In 1999, he joined Delft University of Technology, where he is now an Associate Professor in the Faculty of Electrical Engineering, Computer Science and Mathematics. His main research interests are in the design of precision analog circuitry, sigmadelta modulators and sensor interfaces. This has resulted in ten U.S. patents and over 80 technical papers.

Dr. Makinwa is a Fellow of the Young Academy of the Royal Netherlands Academy of Arts and Sciences. He is on the program committees of several international conferences, including the IEEE International Solid-State Circuits Conference (ISSCC). He has presented several invited talks and tutorials at such conferences, including two at the ISSCC. He is the co-recipient of several best paper awards: JSSC (2005), ISSCC (2008, 2006, 2005), ESSCIRC (2006) and ISCAS (2008). In 2005, he was the recipient of the Veni Award from the Netherlands Organization for Scientific Research and the Simon Stevin Gezel Award from the Dutch Technology Foundation. He is a distinguished lecturer of the IEEE Solid-State Circuits Society.

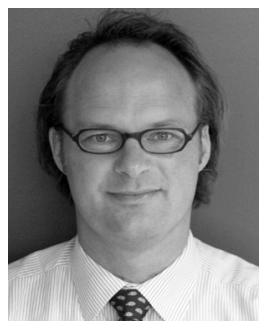

Bram Nauta (F'08) was born in Hengelo, The Netherlands, in 1964. In 1987 he received the M.Sc. degree (cum laude) in electrical engineering and the Ph.D. degree from the University of Twente, Enschede, The Netherlands, in 1987 and 1991, respectively. His doctoral work focused on analog CMOS filters for very high frequencies.

In 1991 he joined the Mixed-Signal Circuits and Systems Department of Philips Research, Eindhoven, The Netherlands, where he worked on high-speed A/D converters and analog key modules. In 1998 he returned to the University of Twente as a full Professor heading the IC Design group, which is part of the CTIT Research Institute. His current research interest is high-speed analog CMOS circuits. In addition he is also a part-time consultant in industry, and in 2001 he cofounded Chip Design Works. His Ph.D. dissertation was published as a book, Analog CMOS Filters for Very High Frequencies (Springer, 1993).

Dr. Nauta was the recipient of the "Shell Study Tour Award" for his Ph.D. work. From 1997 until 1999, he served as an Associate Editor of the IEEE TRANSACTIONS ON CiRCUITS AND SySTEMS-II: ANALOG AND DigitaL SignAL PROCESSING. After this, he served as Guest Editor and Associate Editor (20012006), and since 2007 has been Editor-in-Chief of the IEEE JOURNAL OF SoLIDSTATE CIRCUITS. He is also a member of the technical program committees of the International Solid State Circuits Conference (ISSCC), the European Solid State Circuit Conference (ESSCIRC), and the Symposium on VLSI circuits. He was corecipient of the ISSCC 2002 "Van Vessem Outstanding Paper Award," is a distinguished lecturer of the IEEE, and an elected member of IEEE-SSCS AdCom. 